\title{
Entropic Measure of Time, and Gas Expansion in Vacuum
}

\author{
Leonid M. Martyushev ${ }^{1,2, *}$ and Evgenii V. Shaiapin ${ }^{1}$ \\ 1 Ural Federal University, 19 Mira St., Ekaterinburg 620002, Russia; shayapin@mail.ru \\ 2 Institute of Industrial Ecology, Russian Academy of Sciences, 20 S. Kovalevskaya St., \\ Ekaterinburg 620219, Russia \\ * Correspondence: LeonidMartyushev@gmail.com; Tel.: +7-922-227-7425
}

Academic Editors: Milivoje M. Kostic and Kevin H. Knuth

Received: 29 March 2016; Accepted: 9 June 2016; Published: 21 June 2016

\begin{abstract}
The study considers advantages of the introduced measure of time based on the entropy change under irreversible processes (entropy production). Using the example of non-equilibrium expansion of an ideal gas in vacuum, such a measure is introduced. It is shown that, in the general case, this measure of time proves to be nonlinearly related to the reference measure assumed uniform by convention. The connection between this result and the results of other authors investigating the measure of time in some biological and cosmological problems is noted.
\end{abstract}

Keywords: entropy production; intrinsic and chronological times; ideal gas expansion

\section{Introduction}

The second law of thermodynamics together with the following concept of entropy had a tremendous effect on physics and natural science in general. All these-the introduction of an absolute temperature scale, the restriction of the conversion efficiency of heat engines, the discovery of various regularities under non-equilibrium processes - and many other things originated as crucial corollaries of the second law [1]. The increase of thermodynamic entropy in an isolated system enabled researchers to introduce a physical quantity characterizing irreversibility, an essential property of the world around us that was not previously covered by the laws of mechanics and electromagnetics.

The irreversibility and directionality from the past to the future is a basic property of another physical quantity: time. The problem of time and the properties thereof are addressed by an enormous number of studies, especially philosophic ones. Physicists investigate this quantity much more rarely; however, this area has a number of good reviews (see, for instance, [2-6]). Based on these investigations, we can identify a number of properties that are common for time and entropy. Thus, in addition to directionality, they both are related to variability and depend on an observer. The latter property is more often attributed to Boltzmann's and the subsequent statistical and information generalization of entropy as well as to the most wide-spread approach in the present post-Einstein age: the relational approach to time. Furthermore, both quantities are considered among the fundamental and most difficult-to-study concepts characterizing the surrounding world. It should be mentioned that close relations between entropy and time were first noticed by Boltzmann and then this question was discussed in the literature many times (see, for example, [2,3]).

As regards the noted affinity, there are a number of papers (see, for instance, [7]) where the measurement of time flow is directly associated with the measurement of entropy change (more specifically, with irreversible change, i.e., entropy production). This use of entropy production as a metric of time has a considerable advantage as compared to others. Namely, the traditional methods of introducing a metric of time are based on astronomic, mechanic, electromagnetic, and quantum optical regularities. However, according to the contemporary understanding, they are seen 
as essentially reversible phenomena. Consequently, these regularities are unreasonable from the fundamental perspective, as a basic measure for the essentially irreversible quantity (the so-called arrow of time). Universality is another advantage of entropy-based time. Indeed, the statistical introduction of entropy through a number of microstates enabling a system's state allows finding entropy and, therefore, time for any systems (including imaginary and modeled ones). As opposed to the entropic measure of time, the traditional measures of time are based on some specific phenomenon that a system may lack in the general case (particularly, it is easy to assume a hypothetic world of electrically neutral classic particles where electromagnetic and quantum optical measures of time cannot be used). Thus, the entropy method of introducing a metric of time flow appears to be as universal as the traditional method of introducing a space metric. Moreover, what is very important is that this method is essentially different from the methods associated only with spatial measurements (as distinct, for example, from the employed astronomic and mechanical methods for introducing the measure of time). In addition, the fact that the time considered herein is directly related to a system's dissipative processes and is, therefore, strictly individual for every system involved represents a major feature of this time and its fundamental difference from an absolute Newtonian time.

In spite of the reasons above, the question about relations between entropy and the measure of time has been scarcely studied in the literature. Considerable discussions of this topic from the qualitative perspective can be more often found in philosophic and popular-science editions (see, for instance, $[2-5,8]$ ), while few quantitative works on the topic have been recently presented (see, for instance, [9-12]).

The effort in this area was made in [13]. The metric of time for a developing system $\tau$ was introduced as directly proportional to the specific production of thermodynamic entropy. The found logarithmic relation of $\tau$ to the reference (uniform) time $t$ used by the observer external to the system is an interesting result of such a consideration. A drawback of this research is that the class of systems for which the provided approach is directly suitable is restricted by the postulates of local non-equilibrium thermodynamics. As is known, these restrictions depend on the possibility to consider a system in a local equilibrium state $[1,14]$. The class of non-equilibrium systems which are provided for such an opportunity is very wide (it includes the majority of practically important systems that we encounter). However, there still are systems for which such a consideration is inapplicable [14]. Gas expansion from some finite volume into a vacuumized space of unlimited size represents an important example of such a system. For a number of cases (for instance, under expansion from a thermodynamic equilibrium state, with some restrictions applied to gas properties and expansion rate), this problem can be subjected to hydrodynamic consideration involving thermodynamics. Here, an isoentropic description of the expanding gas (see, for example, [15]) is typically used. Nevertheless, in the extreme case (of the absence of thermodynamic equilibrium in the original state and a lack of interaction of gas molecules with each other and walls during the expansion), this problem falls outside the scope of thermodynamics. According to Popper (see, e.g., $[16,17])$, this example is fundamental as it indicates the presence of unidirectional processes for which entropy either remains unchanged or is not defined. This leads Popper to a conclusion [16,17] that it is incorrect to connect the arrow of time with the increase of entropy and that, starting from Boltzmann, these two notions have been erroneously considered as closely related. However, we think that this conclusion is too categorical. The given example only shows that non-thermodynamic systems cannot be considered with the use of the entropy which was phenomenologically introduced by Clausius and then theoretically developed by Boltzmann for macroscopic systems where interaction between their constituent particles causes relaxation of a system to a thermodynamic equilibrium. If a system fails to meet the basic postulates of thermodynamics [1], then the simplest generalizations of Boltzmann's entropy must be employed. Here, informational entropy is the most well-known and simplest generalization (hereinafter simply referred to as entropy) [3]. It is that which should be related to time in such systems. The measure of time based on such entropy will become applicable to arbitrary systems (not only related to gas expansion, i.e., having a physical origin, but also to systems 
considered in biology, economy, etc.). Based on the above, the important task is to consider systems where the use of classical entropy is restricted, and for such a system it is necessary to find a method of introducing the entropy-based time and analyze the possible corollaries thereof. This subject is the purpose of the present study.

\section{Model}

In order to achieve the stated purpose, we deliberately chose to study a very complicated case in terms of thermodynamic description: expansion of an ideal gas in vacuum, i.e., motion of identical particles that can interact rather rarely only by absolutely elastic collisions. Initially, the gas occupies a certain volume where molecules' locations and velocities are unknown and reasonably arbitrary. The direction of motion is strictly radial. Then the gas starts expanding; during this process, the volume occupied by its molecules increases while the initial distribution of particle velocities, according to the model involved, remains the same. Obviously, any stage of expansion in such a system is not in a thermodynamic equilibrium (including the local one), and thermodynamic equilibrium characteristics such as temperature and the like cannot be introduced. For illustrative purposes, we will consider a one-dimensional case (Figure 1). We note that a three-dimensional case is absolutely similar and will be studied below. Let us assume a number of particles $N$. We have an observer inside the expanding gas with the above properties. The observer would naturally choose a size of one of the system's particles as a length scale. Then other spatial sizes of the problem are normalized to this length scale, i.e., considered dimensionless. These include initial and current sizes of the regions occupied by the gas: $r_{0}$ and $r$, respectively (see Figure 1 ). The observer has no clock (timepiece). However, the purpose of the observer is to introduce a measure of time based on the properties of the system of particles around him. Using the above-mentioned properties of the measure of time and following this paper's purpose, the observer wants to use the entropy method. The entropy that can be introduced for arbitrary, including essentially non-equilibrium, systems [3] would be naturally chosen. For the problem under consideration, the entropy change equals the entropy production (no entropy flows through the system's boundaries). Since only spatial changes occur in the system, the observer divides the system's current size $r$ into $G$ similar cells having the size $\Delta$ (Figure 1). This size remains unchanged and, additionally, can contain up to $N$ particles. It is obvious that, at different system-observation instants, $G$ varies and equals $2 r / \Delta$. The size of the cell selected by the observer depends on the degree of detail in which the observer prefers to describe the system. Based on the information which is known by the observer (or, as we should rather say, a total lack of information about the system, including details of its initial state), all possible distributions of particles by cells should be considered equally probable. This probability is inversely proportional to the number of such distributions $\Omega$. This number by which $N$ identical particles may be arranged by $G$ cells with an arbitrary number of particles per cell is well known [18]:

$$
\Omega=\frac{(N+G-1) !}{N !(G-1) !}
$$

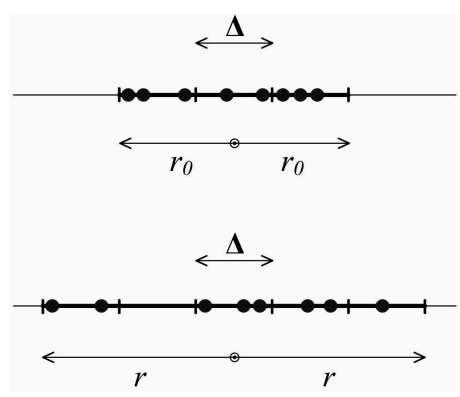

Figure 1. The simplest model of identical particles spreading out in vacuum considered herein. The initial state and one of the following states are shown. All symbols in the figure are explained in the text hereof. 
A similar formula is traditionally used in the literature for describing boson distributions. The difference between the two formulae lies only in their interpretations. Thus, in the case of bosons, cells are primarily associated with energetic rather than spatial states and the number of cells is usually considered constant as opposed to variable in our case.

\section{Entropic Measure of Time}

As noted above, the observer uses such a measure of time that the time change $d \tau$ is equal (accurate up to a constant multiplier) to the irreversible change of entropy per particle $d S_{i r}$ (entropy production density):

$$
d \tau \equiv d S_{i r} / N
$$

In Equation (2), the entropy (which in some known cases is reduced to thermodynamic and Boltzmann entropies) is used and the entropy changes due to irreversible processes inside the system rather than a flow across the system's boundaries. It should be additionally emphasized that the entropic measure (2) has meaning only if irreversible processes take place in the system. In the case of equilibrium, and for hypothetic reversible processes, it loses its meaning.

The entropy $S$ for the system under consideration is defined (accurate up to a constant dimensional multiplier) by [3]:

$$
S=\ln \Omega
$$

Assuming that $N>>1$ and $G>>1$, based on Equations (1) and (3), using Stirling's approximation, we obtain:

$$
S=(N+G) \ln (N+G)-G \ln G-N \ln N
$$

As a result, for entropy per particle we have:

$$
S / N=\left(1+\frac{G}{N}\right) \ln \left(1+\frac{G}{N}\right)-\frac{G}{N} \ln \left(\frac{G}{N}\right)
$$

For the gas expansion problem at hand, obviously $d S_{i r}=d S$. Therefore, using Equation (2), the following can be written, accurate up to an additive constant:

$$
\tau \equiv S / N=\left(1+\frac{G}{N}\right) \ln \left(1+\frac{G}{N}\right)-\frac{G}{N} \ln \left(\frac{G}{N}\right)
$$

The measure of time thus introduced has all the necessary properties. So, for the preset $N$, it increases monotonically under the gas's irreversible expansion which is always accompanied by the increase of $G$. Since $G=2 r / \Delta$, the introduced measure is immediately related to the changes occurring in the system and depends on the observer dividing the space available to the system into similar cells. Furthermore, the introduced measure is rather universal: it requires only an observer (selecting $\Delta$ ), a developing system, and the possibility of introducing the measure of distance.

It is important to establish relations between the introduced measure of time (Equation (6)) and the time $t$ assumed as the reference uniform time. For this purpose, we shall consider an observer positioned outside the system at hand and having such a reference clock. This observer has all the information about the system like the first observer does, i.e., knowledge of the quantities $r_{0}, r, N, G$, and $\Delta$. The outside observer will calculate the system's specific entropy in exactly the same manner as in Equation (5). However, originally having a clock, the observer can introduce the velocity of motion $v=d r / d t$. Therefore, for the considered one-dimensional motion, $G=2\left(r_{0}+v t\right) / \Delta$. As a result, according to Equation (6),

$$
\tau=\left(1+\frac{2\left(r_{o}+v \cdot t\right)}{N \Delta}\right) \ln \left(1+\frac{2\left(r_{o}+v \cdot t\right)}{N \Delta}\right)-\frac{2\left(r_{0}+v \cdot t\right)}{N \Delta} \ln \left(\frac{2\left(r_{o}+v \cdot t\right)}{N \Delta}\right)
$$


Let us introduce the following notation: $\alpha_{1}=\frac{2 r_{0}}{N \Delta}, t_{1}=\frac{N \Delta}{2 v}$. Then we obtain

$$
\tau=\left(1+\alpha_{1}+t / t_{1}\right) \ln \left(1+\alpha_{1}+t / t_{1}\right)-\left(\alpha_{1}+t / t_{1}\right) \ln \left(\alpha_{1}+t / t_{1}\right)
$$

Using Equation (8), we will consider two limits:

- $\quad$ Let $t \rightarrow 0$, then

$$
\tau \propto \tau_{1}+\xi_{1} t / t_{1}
$$

where $\tau_{1}=\left(1+\alpha_{1}\right) \ln \left(1+\alpha_{1}\right)-\alpha_{1} \ln \alpha_{1}, \xi_{1}=\ln \left(1+1 / \alpha_{1}\right)$.

- $\quad$ Let $t \rightarrow \infty$, then

$$
\tau \propto \ln \left(t / t_{1}\right)
$$

The obtained formulae (8)-(10) indicate that, for the general case, the time $\tau$ introduced by the inside observer based on the calculations of entropy is non-uniform (nonlinear) with respect to $t$ (Figure 2). The relation of the two scales appears to be linear only in the very beginning of expansion (see Equation (9)). It may seem that such a property of the entropic measure of time is its drawback. Indeed, it is traditionally assumed in physics that time flows uniformly for velocities much smaller than the velocity of light and for relatively small masses (i.e., outside the scope of the special and general theory of relativity). However, such an assumption is not based on any law; moreover, it cannot be proved either logically or empirically. In particular, this issue was addressed by Poincaré $[19,20]$. Let us here quote his paper [19]:

We have not a direct intuition of the equality of two intervals of time. The persons who believe they possess this intuition are dupes of an illusion. When I say, from noon to one the same time passes as from two to three, what meaning has this affirmation? The least reflection shows that by itself it has none at all. It will only have that which I choose to give it, by a definition which will certainly possess a certain degree of arbitrariness.

And another quotation of his [19]:

... there is not one way of measuring time more true than another; that which is generally adopted is only more convenient. Of two watches, we have no right to say that the one goes true, the other wrong; we can only say that it is advantageous to conform to the indications of the first.

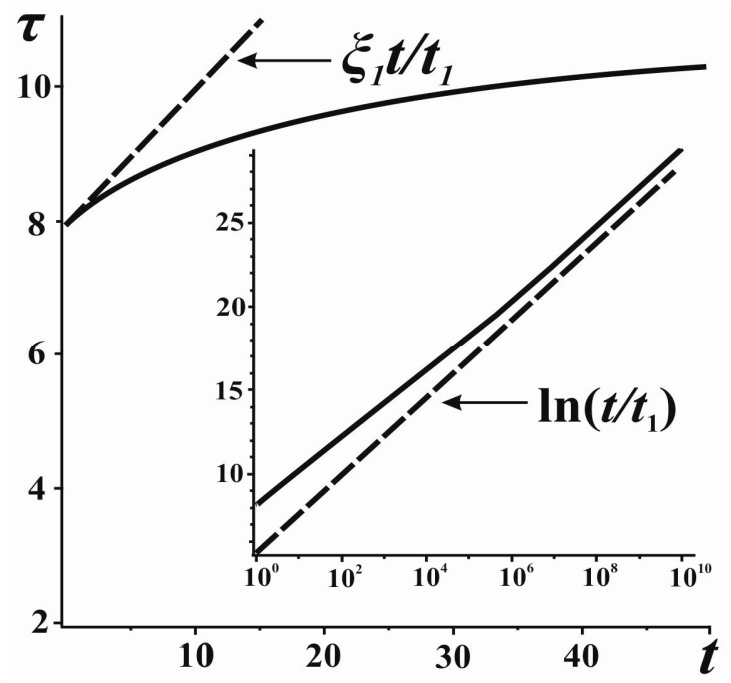

Figure 2. Dependence of the entropic time $\tau$ introduced by the observer inside the system on the time chosen by the outside observer as the uniform time $t$. The dashed lines show limit approximations Equation (11) at small and large times. $t_{1}=0.005, \alpha_{1}=1000$. 
One has to remember that we have assumed the existence of the uniform scale of $t$ that can be used as a reference only by convention. In reality (for instance, from the standpoint of some third observer), the clock of $t$ of the outside observer considered herein may be even more "non-uniform" than that of the inside observer.

Such nonlinearity leads to interesting kinematical corollaries. Indeed, according to the outside observer, the system's boundary expands at a constant velocity $v$, the velocity of motion of the originally fastest particles. However, from the perspective of the inside observer, such constancy of velocity will be observed only in the beginning, and at considerably long times the velocity of motion will grow exponentially. Indeed, according to the inside observer, the velocity $d r / d \tau$ can be written, using Equations (9) and (10), as:

$$
\frac{d r}{d \tau}=\frac{d r}{d t} \frac{d t}{d \tau}=v \frac{d t}{d \tau}=\left\{\begin{array}{cl}
\frac{N \Delta}{2 \xi_{1}}, & t, \tau \rightarrow 0 \\
\frac{N \Delta}{2} e^{\tau}, & t, \tau \rightarrow \infty
\end{array}\right.
$$

As is seen, at some moment of observation, according to the measurements of the inside observer using Newton's dynamics to describe the motion, the particles will start spreading out with an acceleration as if under the influence of a "mysterious" (or "dark") force which arises and becomes stronger with time.

Let us make two important notes.

1. Previously, the one-dimensional case was considered. It can be easily extended to a threedimensional one. The gas expands radially with the spherical symmetry. Initially, it is contained in a sphere with the radius $r_{0}$ and then occupies ever larger spheres of the radius $r$. As before, let us write the number of cells and the number of particles as $G$ and $N$, respectively. As in the case above, we will assume the volume of cells is constant and designate it as $\Delta^{3}$. Given the symmetry of the problem, the shape of the cells can be selected as spherical layers of some thickness centered on the system's point of symmetry. Obviously, the thickness of these layers is to decrease in inverse proportion to $r^{2}$. For such a formulation, $G=4 \pi(r / \Delta)^{3} / 3$. It is evident that the expression for the time measured by the inside observer using the variables $G$ and $N$ will remain the same as Equation (6). However, the relation of the inside observer's time and the reference time has the form:

$$
\tau=\left(1+\left(\alpha_{3}+t / t_{3}\right)^{3}\right) \ln \left(1+\left(\alpha_{3}+t / t_{3}\right)^{3}\right)-3\left(\alpha_{3}+t / t_{3}\right)^{3} \ln \left(\alpha_{3}+t / t_{3}\right)
$$

where $\alpha_{3}=(4 \pi / 3 N)^{1 / 3} r_{0} / \Delta, t_{3}=(3 N / 4 \pi)^{1 / 3} \Delta / v$. Two limit cases have the form:

$$
\tau \propto \tau_{3}+\xi_{3} t / t_{3}, t \rightarrow 0
$$

where $\tau_{3}=\left(1+\alpha_{3}^{3}\right) \ln \left(1+\alpha_{3}^{3}\right)-\alpha_{3}^{3} \ln \left(\alpha_{3}^{3}\right), \xi_{3}=3 \alpha_{3}^{2} \ln \left(1+1 / \alpha_{3}^{3}\right)$.

$$
\tau \propto 3 \ln \left(t / t_{3}\right), t \rightarrow \infty
$$

Thus, as is seen from the given formulae, the three-dimensional case has no fundamental differences from the linear one.

2. Previously, we have considered a strongly non-equilibrium case of gas expansion in vacuum. This case cannot be investigated using the methods of classical thermodynamics. It can be shown, nevertheless, that for a number of adjacent problems, results similar to the ones above can be obtained thermodynamically using a number of restrictions. We will consider some initial thermodynamic equilibrium state of an ideal gas. The gas adiabatically expands from it in vacuum and reaches another thermodynamic equilibrium state while changing the volume $V$. Obviously, this process is irreversible and the gas does no work. According to the adiabatic 
nature of the process and the first law of thermodynamics, the temperature of an ideal gas is to remain unchanged during expansion. Let us replace a real irreversible process with a hypothetic isothermal equilibrium process having identical initial and final states. The changes of thermodynamic entropy $S_{t}$ for the two processes are the same and it is easy to show $[1,18]$ that the change of entropy in the case at hand is equal to

$$
d S_{t}=v R d V / V
$$

where $R$ is the universal gas constant and $v$ is the number of gas moles.

As before, by introducing the inside observer's time as directly proportional to entropy per particle, we obtain

$$
d \tau \propto d S_{t} / v
$$

or, with the accuracy up to an additive constant and multipliers,

$$
\tau=\ln V
$$

For the outside observer (having the reference uniform clock of $t$ ), the gas volume during expansion is described by a power-law dependence on $t$. By inserting this law into the last formula, a logarithmic relation between the two times, similar to Equation (14), is obtained.

\section{Conclusions}

The present study develops a formerly proposed relation between the measure of time and entropy. The thermodynamic entropy previously used for this purpose in the case of local-equilibrium processes is replaced with the informational entropy suitable for describing arbitrary systems. For the simplest model of ideal-gas expansion, we obtain the entropic measure of time related to the spatial disorder.

It was not an intention hereof to answer metaphysical questions about the cause of variability in the world, the nature of time, its reality, and the like. We considered a purely physical problem of the most consistent and universal, from the theoretical point of view, introduction of the measure of time. Such a rigorous operational approach to the introduction of measures (particularly the measure of time) of the basic physical quantities is extremely important for the foundation of physics and its further development. It was mentioned many times by Bridgman [21], and Milne [22], et al.

Importantly, it is the existence of a special function of state, entropy (i.e., one of the formulations of the second law of thermodynamics), that allowed introducing an absolute temperature scale for a thermodynamic system in a thermal equilibrium. As a result, an objective measure of thermal-motion intensity appeared in science [1]. The present study connects the change of entropy under irreversible processes with the possibility to consistently introduce the measure of time. The noted relations between entropy, on the one side, and temperature/time, on the other side, may prove to be very meaningful and require an additional research.

The crucial result hereof is that a nonlinear relation between the time scale used by an observer and the one chosen as a reference is established for the general case. We believe that a logarithmic relation observed at relatively long times is especially important here (see Equation (14)). To be specific, such a relation between intrinsic (developmental) and chronological (astronomical) times during growth and development of systems was independently proposed before $[13,23,24]$. In particular, the papers $[13,24]$ provide a theoretical grounding for the universal power-law relationship between a developing system's mass and astronomical time. This relationship is confirmed by the available empirical data of the growth in biological and crystallization systems. Then the change of mass in the system is connected with its entropy production which, similarly to the approach herein, is directly related to the internal (biological) time in the system. Additionally, such a logarithmic relation between the two scales of time and its potential significance for cosmology was also mentioned in 1937-1950s by Milne (see, for instance, [22]). It is interesting that Milne obtained this relation only 
kinematically while bringing his laws of motion, obtained for the so-called fundamental particles (galactic nuclei), into consistency with the traditional Newton laws. The fundamental particles formed a basis of his cosmological model of the world. These particles spread out uniformly in different directions at different velocities (largely like the expansion of an ideal gas in vacuum considered herein). Milne shows that the uniform motion of the fundamental particles occurs for time $t$ (a universal time related to the atomic clock), whereas $\tau$ (which is logarithmically associated with $t$ ) is a time introduced by the researcher on the basis of astronomic observations. Such use of the two times allowed Milne to build an original theory that had a great influence on the development of modern cosmology.

The parallels mentioned herein obviously demonstrate a common nature and a close connection between the measure of time considered in the present paper and the measures of time used both in the problems of cosmology to describe the origin and development of the Universe and in the problems of biology to describe the birth and growth of living beings.

Despite the result obtained herein with respect to the logarithmic relation between the measures of time and its mentioned connection with the results of other studies, the question of universality of such a relation requires further serious analysis and, presently, generalizations (in particular regarding cosmological problems) must be avoided.

Acknowledgments: The authors express their special thanks to Vladimir D. Seleznev for discussion and unfailing interest to the topic considered herein. His death on 12 October 2015 became a tremendous personal tragedy for us. His memory will always be with us. We dedicate this research on entropy and time to him. Vladimir D. Seleznev contributed the most of his time on this earth to the study of entropy and his ideas will dwell with us for a very long time. The reported study was partially funded by RFBR according to the research project No. 16-31-00255 MOA_a and one of the authors (Evgenii V. Shaiapin) expresses his gratitude for it.

Author Contributions: Leonid M. Martyushev proposed all the basic ideas of the research including the problem of calculation of the entropic measure of time for an ideal gas expanding in vacuum. He also wrote the basic text of the paper. Evgenii V. Shaiapin carried out all the necessary calculations and prepared the manuscript for publication. Both authors analyzed and discussed the results, read and approved the final manuscript.

Conflicts of Interest: The authors declare no conflict of interest.

\section{References}

1. Bazarov, I.P. Thermodynamics; Pergamon Press: New York, NY, USA, 1964.

2. Whitrow, G.J. The Natural Philosophy of Time; Oxford University Press: Oxford, UK, 1960.

3. Reichenbach, H. The Direction of Time; University of California Press: Berkeley, CA, USA, 1991.

4. Hawking, S. A Brief History of Time; Bantam Books: New York, NY, USA, 1988.

5. Landsberg, P.T. Irreversibility and Time's Arrow. Dialectica 1996, 50, 247-258. [CrossRef]

6. Halliwell, J.J.; Pérez-Mercader, J.; Zurek, W.H. Physical Origins of Time Asymmetry; Cambridge University Press: Cambridge, UK, 1996.

7. Richardson, I.W.; Rosen, R. Aging and the metrics of time. J. Theor. Biol. 1979, 79, 415-423. [CrossRef]

8. Prigogine, I.; Stengers, I. Order out of Chaos: Man's New Dialogue with Nature; Heinemann: London, UK, 1984.

9. Haddad, W.M. Temporal Asymmetry, Entropic Irreversibility, and Finite-Time Thermodynamics: From Parmenides-Einstein Time-Reversal Symmetry to the Heraclitan Entropic Arrow of Time. Entropy 2012, 14, 407-455. [CrossRef]

10. Feng, E.H.; Crooks, G.E. Length of Time's Arrow. Phys. Rev. Lett. 2008, 101, 090602. [CrossRef] [PubMed]

11. Roldán, E.; Neri, I.; Dörpinghaus, M.; Meyr, H.; Jülicher, F. Decision Making in the Arrow of Time. Phys. Rev. Lett. 2015, 115, 250602. [CrossRef] [PubMed]

12. Sasa, S.-I.; Yokokura, Y. Thermodynamic entropy as a Noether invariant. Phys. Rev. Lett. 2016, $116,140601$. [CrossRef] [PubMed]

13. Martyushev, L.M.; Terentiev, P.S. A universal model of ontogenetic growth. Sci. Nat. 2015, 102, 29. [CrossRef] [PubMed]

14. Lebon, G.; Jou, D.; Casas-Vázquez, J. Understanding Non-Equilibrium Thermodynamics: Foundations, Applications, Frontiers; Springer: Berlin, Germany; Heidelberg, Germany, 2008.

15. Zel'dovich, Y.B.; Raizer, Y.P. Physics of Shock Waves and High-Temperature Hydrodynamic Phenomena; Dover: Mineola, NY, USA, 2002. 
16. Popper, K.R. Reply to R. Schlegel. Nature 1956, 178, 382-384. [CrossRef]

17. Popper, K.R. Reply to R. C. L. Bosworth. Nature 1958, 181, 402-403. [CrossRef]

18. Landau, L.D.; Lifshitz, E.M. Statistical Physics; Butterworth-Heinemann: Oxford, UK, 1980.

19. Poincaré, H. The Measure of Time (Engl. Transl.). In The Foundations of Science; Science Press: New York, NY, USA, 1913; pp. 222-234, Rev. Métaphys. Morale 1898, 6, 1-13.

20. Poincaré, H. Last Essays; Dover: New York, NY, USA, 1963.

21. Bridgman, P. Einstein's theories and the operational point of view. In Albert Einstein: Philosopher-Scientist; Schlipp, P.A., Ed.; Open Court: Evanston, IL, USA, 1949; pp. 335-354.

22. Milne, E.A. Kinematic Relativity; Clarendon Press: Oxford, UK, 1948.

23. Backman, G. Wachstum und Organische Zeit; Barth: Leipzig, Germany, 1943. (In German)

24. Martyushev, L.M.; Sergeev, A.P.; Terentiev, P.S. Invariance of specific mass increment in the case of non-equilibrium growth. Chin. Phys. B 2015, 24, 090502. [CrossRef]

(C) 2016 by the authors; licensee MDPI, Basel, Switzerland. This article is an open access article distributed under the terms and conditions of the Creative Commons Attribution (CC-BY) license (http://creativecommons.org/licenses/by/4.0/). 